\title{
Madridge
}

\section{madridge Journal of Novel Drug Research}

Editorial Article

Open Access

\section{Adamantane, Irene, Naftazone and Pyridine-Enhanced Precatalyst Preparation Stabilization and Initiation (PEPPSI) Nano Molecules}

\author{
Alireza Heidari* \\ Faculty of Chemistry, California South University, 14731 Comet St. Irvine, CA 92604, USA
}

\section{Article Info}

*Corresponding author:

Alireza Heidari

California South University

14731 Comet St. Irvine

CA 92604

USA

Email:

Scholar.Researcher.Scientist@gmail.com;

Alireza.Heidari@calsu.us

\section{Received: May 26, 2018}

Accepted: July 17, 2018

Published: July 23, 2018

Citation: Heidari A. Adamantane, Irene, Naftazone and Pyridine-Enhanced Precatalyst Preparation Stabilization and Initiation (PEPPSI) Nano Molecules. Madridge J Nov Drug Res. 2018; 2(1): 61-67.

doi: $10.18689 / \mathrm{mjndr}-1000109$

Copyright: (c) 2018 The Author(s). This work is licensed under a Creative Commons Attribution 4.0 International License, which permits unrestricted use, distribution, and reproduction in any medium, provided the original work is properly cited.

Published by Madridge Publishers
Keywords: Polymer; Precatalyst preparation; Synchrocyclotron Radiations; Pyridine.

\section{Editorial Article}

In the current editorial, we study Adamantane, Irene, Naftazone and Pyridineenhanced precatalyst preparation stabilization and initiation (PEPPSI) Nano molecules (Figure 1) incorporation into the Nano Polymeric Matrix (NPM) by immersion of the Nano Polymeric Modified Electrode (NPME) as molecular enzymes and drug targets for human cancer cells, Tissues and tumors treatment under synchrotron and synchrocyclotron radiations. In this regard, the development of Chemical Modified Electrodes (CEMs) is at present an area of great interest. CEMs can be divided broadly into two main categories; namely, Surface modified and bulk modified electrodes. Methods of surface modification include adsorption, Covalent bonding, attachment of polymer Nano films, etc. Polymer Nano film coated electrodes can be differentiated from other modification methods such as adsorption and covalent bonding in that they usually involve multilayer as opposed to monolayer frequently encountered for the latter methods. The thicker Nano films imply more active sites which lead to larger analytical signals. This advantage coupled with other, Their versatility and wide applicability, Makes polymer Nano film modified electrodes particularly suitable for analytical applications [1-27].

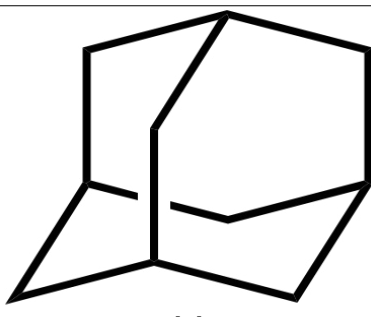

(a)

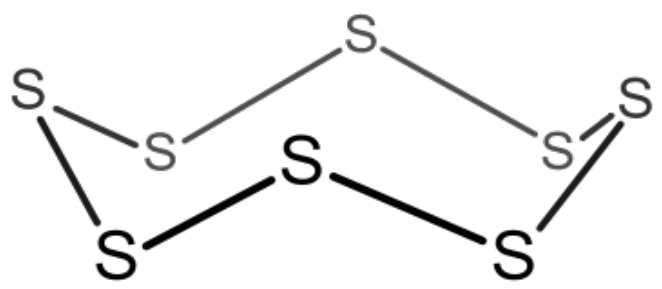

(b)<smiles></smiles>

(c)

(d)

Figure 1. Molecular structure of (a) Adamantane, (b) Irene, (c) Naftazone and (d) PEPPSI Nano molecules. 
Electrochemical polymerization offers the advantage of reproducible deposition in terms of Nano film thickness and loading, Making the immobilization procedure of a metalbased electrocatalyst very simple and reliable for Adamantane, Irene, Naftazone and Pyridine-enhanced precatalyst preparation stabilization and initiation (PEPPSI) Nano molecules-encapsulating Carbon nanotubes incorporation into the Nano Polymeric Matrix (NPM) by immersion of the Nano Polymeric Modified Electrode (NPME) as molecular enzymes and drug targets for human cancer cells, Tissues and tumors treatment under synchrotron and synchrocyclotron radiations, Also it must be notice that the nature of working electrode substrate in electropreparation of polymeric Nano film is very important, Because properties of polymeric Nano films depend on the working electrode anti-cancer Nano materials. The ease and fast preparation and of obtaining a new reproducible surface, The low residual current, porous surface and low cost of Multi-Walled Carbon Nanotubes (MWCNTs) paste are some advantages of Carbon Paste Electrode (CPE) over all other solid electrodes [28-92].

On the other hand, it has been shown that, macrocyclic complexes of Adamantane, Irene, Naftazone and Pyridineenhanced precatalyst preparation stabilization and initiation (PEPPSI) Nano molecules-encapsulating Carbon nanotubes are interest as modifying agents because in basic media Adamantane, Irene, Naftazone and Pyridine-enhanced precatalyst preparation stabilization and initiation (PEPPSI) Nano molecules-encapsulating Carbon nanotubes redox centers show high catalytic activity towards the oxidation of small organic anti-cancer Nano compounds. The high-valence species of Adamantane, Irene, Naftazone and Pyridineenhanced precatalyst preparation stabilization and initiation (PEPPSI) Nano molecules-encapsulating Carbon nanotubes seem to act as strong oxidizing agents for low-electroactivity organic substrates. 1,2-Dioxetane (1,2-Dioxacyclobutane), 1,3-Dioxetane (1,3-Dioxacyclobutane), DMDM Hydantoin and Sulphobe as the anti-cancer organic intermediate products of methanol oxidation as well as formic acid, is important to investigate its electrochemical oxidation behavior in Adamantane, Irene, Naftazone and Pyridine-enhanced precatalyst preparation stabilization and initiation (PEPPSI) Nano molecules-encapsulating Carbon nanotubes incorporation into the Nano Polymeric Matrix (NPM) by immersion of the Nano Polymeric Modified Electrode (NPME) as molecular enzymes and drug targets for human cancer cells, Tissues and tumors treatment under synchrotron and synchrocyclotron radiations [93-110].

In this editorial, we decided to combine the above mentioned advantageous features for the aim of Adamantane, Irene, Naftazone and Pyridine-enhanced precatalyst preparation stabilization and initiation (PEPPSI) Nano moleculesencapsulating Carbon nanotubes incorporation into the Nano Polymeric Matrix (NPM) by immersion of the Nano Polymeric Modified Electrode (NPME) as molecular enzymes and drug targets for human cancer cells, Tissues and tumors treatment under synchrotron and synchrocyclotron radiations. Furthermore, in this editorial, we prepared poly Nano films by electropolymerization at the surface of Multi-Walled Carbon Nanotubes (MWCNTs) paste electrode. Then, Adamantane, Irene, Naftazone and Pyridine-enhanced precatalyst preparation stabilization and initiation (PEPPSI) Nano molecules-encapsulating Carbon nanotubes were incorporated into the Nano Polymeric Matrix (NPM) by immersion of the Nano Polymeric Modified Electrode (NPME) in a solution. The modifier layer of Adamantane, Irene, Naftazone and Pyridine-enhanced precatalyst preparation stabilization and initiation (PEPPSI) Nano moleculesencapsulating Carbon nanotubes at the electrode surface acts as a Nano catalyst for the treatment of human cancer cells, tissues and tumors under synchrotron and synchrocyclotron radiations. Suitability of this Adamantane, Irene, Naftazone and Pyridine-enhanced precatalyst preparation stabilization and initiation (PEPPSI) Nano molecules-encapsulating Carbon nanotubes-modified polymeric Multi-Walled Carbon Nanotubes (MWCNTs) paste electrode toward the electrocatalytic treatment of human cancer cells, tissues and tumors under synchrotron and synchrocyclotron radiations in alkaline medium at ambient temperature was investigated [111-150].

\section{References}

1. Heidari A. Brown C. Study of Composition and Morphology of Cadmium Oxide (CdO) Nanoparticles for Eliminating Cancer Cells. Journal of Nanomedicine Research. 2015; 2(5): 20.

2. Heidari A. Brown C. Study of Surface Morphological. Phytochemical and Structural Characteristics of Rhodium (III) Oxide $\left(\mathrm{Rh}_{2} \mathrm{O}_{3}\right)$ Nanoparticles. International Journal of Pharmacology. Phytochemistry and Ethnomedicine. 2015; 1: 15-19. doi: 10.18052/www.scipress.com/IJPPE.1.15

3. Heidari A. An Experimental Biospectroscopic Study on Seminal Plasma in Determination of Semen Quality for Evaluation of Male Infertility. Int J Adv Technol. 2016; 7(007). doi: 10.4172/0976-4860.1000e007

4. Heidari A. Extraction and Preconcentration of N-Tolyl-SulfonylPhosphoramid-Saeure-Dichlorid as an Anti-Cancer Drug from Plants. A Pharmacognosy Study. J Pharmacogn Nat Prod. 2016; 2(103). doi: 10.4172/2472-0992.1000e103

5. Heidari A. A Thermodynamic Study on Hydration and Dehydration of DNA and RNA-Amphiphile Complexes. J Bioeng Biomed Sci S. 2016; 006. doi: 10.4172/2155-9538.S3-006

6. Heidari A. Computational Studies on Molecular Structures and Carbonyl and Ketene Groups' Effects of Singlet and Triplet Energies of Azidoketene $\mathrm{O}=\mathrm{C}=\mathrm{CH}-\mathrm{NNN}$ and Isocyanatoketene $\mathrm{O}=\mathrm{C}=\mathrm{CH}-\mathrm{N}=\mathrm{C}=\mathrm{O}$. J Appl Computat Math. 2016; 5(142). doi: 10.4172/2168-9679.1000e142

7. Heidari A. Study of Irradiations to Enhance the Induces the Dissociation of Hydrogen Bonds between Peptide Chains and Transition from Helix Structure to Random Coil Structure Using ATR-FTIR. Raman and ${ }^{1} \mathrm{HNMR}$ Spectroscopies. J Biomol Res Ther. 2016; 5(146). doi: 10.4172/21677956.1000e146

8. Heidari A. Future Prospects of Point Fluorescence Spectroscopy. Fluorescence Imaging and Fluorescence Endoscopy in Photodynamic Therapy (PDT) for Cancer Cells. J Bioanal Biomed. 2016; 8(135). doi: 10.4172/1948-593X.1000e135

9. Heidari A. A Bio-Spectroscopic Study of DNA Density and Color Role as Determining Factor for Absorbed Irradiation in Cancer Cells. Adv Cancer Prev. 2013; 1(102). doi: 10.4172/acp.1000e102

10. Heidari A. Manufacturing Process of Solar Cells Using Cadmium Oxide (CdO) and Rhodium (III) Oxide $\left(\mathrm{Rh}_{2} \mathrm{O}_{3}\right)$ Nanoparticles. J Biotechnol Biomater. 2016; 6(125) 2016. doi: 10.4172/2155-952X.1000e125

11. Heidari A. A Novel Experimental and Computational Approach to Photobiosimulation of Telomeric DNA/RNA: A Biospectroscopic and Photobiological Study. J Res Development. 2016; 4(144). doi: 10.4172/2311-3278.1000144 
12. Heidari A. Biochemical and Pharmacodynamical Study of Microporous Molecularly Imprinted Polymer Selective for Vancomycin. Teicoplanin. Oritavancin. Telavancin and Dalbavancin Binding. Biochem Physiol. 2016; 5(146). doi: 10.4172/2168-9652.1000e146

13. Heidari A. Anti-Cancer Effect of UV Irradiation at Presence of Cadmium Oxide (CdO) Nanoparticles on DNA of Cancer Cells, A Photodynamic Therapy Study. Arch Cancer Res. 2016; 4(1).

14. Heidari A. Biospectroscopic Study on Multi-Component Reactions (MCRs) in Two A-Type and B-Type Conformations of Nucleic Acids to Determine Ligand Binding Modes. Binding Constant and Stability of Nucleic Acids in Cadmium Oxide (CdO) Nanoparticles-Nucleic Acids Complexes as AntiCancer Drugs. Arch Cancer Res. 2016; 4(2).

15. Heidari A. Simulation of Temperature Distribution of DNA/RNA of Human Cancer Cells Using Time-Dependent Bio-Heat Equation and Nd: YAG Lasers. Arch Cancer Res. 2016; 4(2).

16. Heidari A. Quantitative Structure-Activity Relationship (QSAR) Approximation for Cadmium Oxide (CdO) and Rhodium (III) Oxide $\left(\mathrm{Rh}_{2} \mathrm{O}_{3}\right)$ Nanoparticles as Anti-Cancer Drugs for the Catalytic Formation of Proviral DNA from Viral RNA Using Multiple Linear and Non-Linear Correlation Approach. Ann Clin Lab Res. 2016; 4(1).

17. Heidari A. Biomedical Study of Cancer Cells DNA Therapy Using Laser Irradiations at Presence of Intelligent Nanoparticles. J Biomedical Sci. 2016; 5(2). doi: 10.4172/2254-609X.100023

18. Heidari A. Measurement the Amount of Vitamin D2 (Ergocalciferol). Vitamin D3 (Cholecalciferol) and Absorbable Calcium (Ca $\left.{ }^{2+}\right)$. Iron (II) $\left(\mathrm{Fe}^{2+}\right)$. Magnesium $\left(\mathrm{Mg}^{2+}\right)$. Phosphate $\left(\mathrm{PO}^{4-}\right)$ and Zinc $\left(\mathrm{Zn}^{2+}\right)$ in Apricot Using High-Performance Liquid Chromatography (HPLC) and Spectroscopic Techniques. J Biom Biostat. 2016; 7(292). doi: 10.4172/2155-6180.1000292

19. Heidari A. Spectroscopy and Quantum Mechanics of the Helium Dimer $\left(\mathrm{He}^{2+}\right)$. Neon Dimer $\left(\mathrm{Ne}^{2+}\right)$. Argon Dimer $\left(\mathrm{Ar}^{2+}\right)$. Krypton Dimer $\left(\mathrm{Kr}^{2+}\right)$. Xenon Dimer $\left(\mathrm{Xe}^{2+}\right)$. Radon Dimer $\left(\mathrm{Rn}^{2+}\right)$ and Ununoctium Dimer $\left(\mathrm{Uuo}^{2+}\right)$ Molecular Cations. Chem Sci J. 2016; 7(112). doi: 10.4172/21503494.1000e112

20. Heidari A. Human Toxicity Photodynamic Therapy Studies on DNA/RNA Complexes as a Promising New Sensitizer for the Treatment of Malignant Tumors Using Bio-Spectroscopic Techniques. J Drug Metab Toxicol. 2016; 7(129). doi: 10.4172/2157-7609.1000e129

21. Heidari A. Novel and Stable Modifications of Intelligent Cadmium Oxide (CdO) Nanoparticles as Anti-Cancer Drug in Formation of Nucleic Acids Complexes for Human Cancer Cells' Treatment. Biochem Pharmacol. 2016; 5(207). doi: 10.4172/2167-0501.1000207

22. Heidari A. A Combined Computational and QM/MM Molecular Dynamics Study on Boron Nitride Nanotubes (BNNTs). Amorphous Boron Nitride Nanotubes (a-BNNTs) and Hexagonal Boron Nitride Nanotubes (hBNNTs) as Hydrogen Storage. Struct Chem Crystallogr Commun. 2016; 2(1).

23. Heidari A. Pharmaceutical and Analytical Chemistry Study of Cadmium Oxide (CdO) Nanoparticles Synthesis Methods and Properties as AntiCancer Drug and its Effect on Human Cancer Cells. Pharm Anal Chem Open Access. 2016; 2(113). doi: 10.4172/2471-2698.1000113

24. Heidari A. A Chemotherapeutic and Biospectroscopic Investigation of the Interaction of Double-Standard DNA/RNA-Binding Molecules with Cadmium Oxide (CdO) and Rhodium (III) Oxide $\left(\mathrm{Rh}_{2} \mathrm{O}_{3}\right)$ Nanoparticles as Anti-Cancer Drugs for Cancer Cells' Treatment. Chemo Open Access. 2016; 5(129). doi: 10.4172/2167-7700.1000e129

25. Heidari A. Pharmacokinetics and Experimental Therapeutic Study of DNA and Other Biomolecules Using Lasers: Advantages and Applications. J Pharmacokinet Exp Ther. 2016; 1(005). doi: 10.4172/jpet.1000e005

26. Heidari A. Determination of Ratio and Stability Constant of DNA/RNA in Human Cancer Cells and Cadmium Oxide (CdO) Nanoparticles Complexes Using Analytical Electrochemical and Spectroscopic Techniques. Insights Anal Electrochem. 2016; 2(1).

27. Heidari A. Discriminate between Antibacterial and Non-Antibacterial Drugs Artificial Neutral Networks of a Multilayer Perceptron (MLP) Type Using a Set of Topological Descriptors. J Heavy Met Toxicity Dis. 2016; $1(2)$.

Madridge J Nov Drug Res.

Volume 2 • Issue $1 \cdot 1000109$
28. Heidari A. Combined Theoretical and Computational Study of the Belousov-Zhabotinsky Chaotic Reaction and Curtius Rearrangement for Synthesis of Mechlorethamine. Cisplatin. Streptozotocin. Cyclophosphamide. Melphalan. Busulphan and BCNU as Anti-Cancer Drugs. Insights Med Phys. 2016; 1(2). doi: 10.21767/2574-285X.100007

29. Heidari A. A Translational Biomedical Approach to Structural Arrangement of Amino Acids' Complexes: A Combined Theoretical and Computational Study. Transl Biomed. 2016; 7(2)

30. Heidari A. Ab Initio and Density Functional Theory (DFT) Studies of Dynamic NMR Shielding Tensors and Vibrational Frequencies of DNA/ RNA and Cadmium Oxide (CdO) Nanoparticles Complexes in Human Cancer Cells. J Nanomedine Biotherapeutic Discov. 2016; 6(144). doi: 10.4172/2155-983X.1000e144

31. Heidari A. Molecular Dynamics and Monte-Carlo Simulations for Replacement Sugars in Insulin Resistance. Obesity. LDL Cholesterol. Triglycerides. Metabolic Syndrome. Type 2 Diabetes and Cardiovascular Disease: A Glycobiological Study. J Glycobiol. 2016; 5(111). doi: 10.4172/2168-958X.1000e111

32. Heidari A. Synthesis and Study of 5-[(Phenylsulfonyl)Amino]-1.3.4Thiadiazole-2-Sulfonamide as Potential Anti-Pertussis Drug Using Chromatography and Spectroscopy Techniques. Transl Med (Sunnyvale). 2016; 6(138). doi: 10.4172/2161-1025.1000e137

33. Heidari A. Nitrogen. Oxygen. Phosphorus and Sulphur Heterocyclic AntiCancer Nano Drugs Separation in the Supercritical Fluid of Ozone $\left(\mathrm{O}_{3}\right)$ Using Soave-Redlich-Kwong (SRK) and Pang-Robinson (PR) Equations. Electronic J Biol. 2016; 12(4)

34. Heidari A. An Analytical and Computational Infrared Spectroscopic Review of Vibrational Modes in Nucleic Acids. Austin J Anal Pharm Chem. 2016; 3(1): 1058

35. Heidari A, Brown C. Phase, Composition and Morphology Study and Analysis of Os-Pd/HfC Nanocomposites. Nano Res Appl.2016; 2(1).

36. Heidari A. Brown C. Vibrational Spectroscopic Study of Intensities and Shifts of Symmetric Vibration Modes of Ozone Diluted by Cumene. International Journal of Advanced Chemistry. 2016; 4 (1): 5-9. doi: 10.14419/ijac.v4i1.6080

37. Heidari A. Study of the Role of Anti-Cancer Molecules with Different Sizes for Decreasing Corresponding Bulk Tumor Multiple Organs or Tissues. Arch Can Res. 2016; 4(2)

38. Heidari A. Genomics and Proteomics Studies of Zolpidem. Necopidem. Alpidem. Saripidem. Miroprofen. Zolimidine. Olprinone and Abafungin as Anti-Tumor. Peptide Antibiotics. Antiviral and Central Nervous System (CNS) Drugs. J Data Mining Genomics \& Proteomics. 2016; 7(125). doi: 10.4172/2153-0602.1000e125

39. Heidari A. Pharmacogenomics and Pharmacoproteomics Studies of Phosphodiesterase-5 (PDE5) Inhibitors and Paclitaxel Albumin-Stabilized Nanoparticles as Sandwiched Anti-Cancer Nano Drugs between Two DNA/RNA Molecules of Human Cancer Cells. J Pharmacogenomics Pharmacoproteomics. 2016; 7(153). doi: 10.4172/2153-0645.1000e153

40. Heidari A. Biotranslational Medical and Biospectroscopic Studies of Cadmium Oxide (CdO) Nanoparticles-DNA/RNA Straight and Cycle Chain Complexes as Potent Anti-Viral. Anti-Tumor and Anti-Microbial Drugs: A Clinical Approach. Transl Biomed. 2016; 7(2).

41. Heidari A. A Comparative Study on Simultaneous Determination and Separation of Adsorbed Cadmium Oxide (CdO) Nanoparticles on DNA/ RNA of Human Cancer Cells Using Biospectroscopic Techniques and Dielectrophoresis (DEP) Method. Arch Can Res. 2016; 4(2).

42. Heidari A. Cheminformatics and System Chemistry of Cisplatin, Carboplatin, Nedaplatin, Oxaliplatin, Heptaplatin and Lobaplatin as AntiCancer Nano Drugs: A Combined Computational and Experimental Study. J Inform Data Min. 2016; 1(3). doi: 10.21767/2472-1956.100015

43. Heidari A. Linear and Non-Linear Quantitative Structure-Anti-CancerActivity Relationship (QSACAR) Study of Hydrous Ruthenium (IV) Oxide (RuO2) Nanoparticles as Non-Nucleoside Reverse Transcriptase Inhibitors (NNRTIs) and Anti-Cancer Nano Drugs. J Integr Oncol. 2016; 5(110). doi: 10.4172/2329-6771.1000e110 
44. Heidari A. Synthesis. Characterization and Biospectroscopic Studies of Cadmium Oxide ( $\mathrm{CdO}$ ) Nanoparticles-Nucleic Acids Complexes Absence of Soluble Polymer as a Protective Agent Using Nucleic Acids Condensation and Solution Reduction Method. J Nanosci Curr Res. 2016; 1(101). doi: 10.4172/2572-0813.1000e101

45. Heidari A. Coplanarity and Collinearity of 4'-Dinonyl-2.2'-Bithiazole in One Domain of Bleomycin and Pingyangmycin to be Responsible for Binding of Cadmium Oxide (CdO) Nanoparticles to DNA/RNA Bidentate Ligands as Anti-Tumor Nano Drug. Int J Drug Dev \& Res. 2016; 8: 007-008.

46. A. A Pharmacovigilance Study on Linear and Non-Linear Quantitative Structure (Chromatographic) Retention Relationships (QSRR) Models for the Prediction of Retention Time of Anti-Cancer Nano Drugs under Synchrotron Radiations. J Pharmacovigil. 2015; 4(161). doi: 10.4172/23296887.1000e161

47. Heidari A. Nanotechnology in Preparation of Semipermeable Polymers. J Adv Chem Eng. 2016; 6 (157).

48. Heidari A. A Gastrointestinal Study on Linear and Non-Linear Quantitative Structure (Chromatographic) Retention Relationships (QSRR) Models for Analysis 5-Aminosalicylates Nano Particles as Digestive System Nano Drugs under Synchrotron Radiations. J Gastrointest Dig Syst. 2016; 6(119). doi: 10.4172/2161-069X.1000e119

49. Heidari A. DNA/RNA Fragmentation and Cytolysis in Human Cancer Cells Treated with Diphthamide Nano Particles Derivatives. Biomedical Data Mining. 2016; 5(102). doi: 10.4172/2090-4924.1000e102

50. Heidari A. A Successful Strategy for the Prediction of Solubility in the Construction of Quantitative Structure-Activity Relationship (QSAR) and Quantitative Structure-Property Relationship (QSPR) under Synchrotron Radiations Using Genetic Function Approximation (GFA) Algorithm. J Mol Biol Biotechnol. 2016; 1(1).

51. Heidari A. Computational Study on Molecular Structures of C20. C60. C240. C540. C960. C2160 and C3840 Fullerene Nano Molecules under Synchrotron Radiations Using Fuzzy Logic. J Material Sci Eng. 2016; 5(282). doi: 10.4172/2169-0022.1000282

52. Heidari A. Graph Theoretical Analysis of Zigzag Polyhexamethylene Biguanide. Polyhexamethylene Adipamide. Polyhexamethylene Biguanide Gauze and Polyhexamethylene Biguanide Hydrochloride (PHMB) Boron Nitride Nanotubes (BNNTs). Amorphous Boron Nitride Nanotubes (aBNNTs) and Hexagonal Boron Nitride Nanotubes (h-BNNTs). J Appl Computat Math. 2016; 5(143). doi: 10.4172/2168-9679.1000e143

53. Heidari A. The Impact of High Resolution Imaging on Diagnosis. Int J Clin Med Imaging. 2016; 39(6): 1000-101. doi: 10.4172/2376-0249.1000e101

54. Heidari A. A Comparative Study of Conformational Behavior of Isotretinoin (13-Cis Retinoic Acid) and Tretinoin (All-Trans Retinoic Acid (ATRA)) Nano Particles as Anti-Cancer Nano Drugs under Synchrotron Radiations Using Hartree-Fock (HF) and Density Functional Theory (DFT) Methods. Insights in Biomed. 2016; 1(2).

55. Heidari A. Advances in Logic. Operations and Computational Mathematics. J Appl Computat Math. 2016; 5(5).

56. Heidari A. Mathematical Equations in Predicting Physical Behavior. J Appl Computat Math. 2016; 5(5). doi: 10.4172/2168-9679.1000e145

57. Heidari A. Chemotherapy a Last Resort for Cancer Treatment. Chemo Open Access. 2016; 5(4). doi: 10.4172/2167-7700.1000e130

58. Heidari A. Separation and Pre-Concentration of Metal Cations-DNA/RNA Chelates Using Molecular Beam Mass Spectrometry with Tunable Vacuum Ultraviolet (VUV) Synchrotron Radiation and Various Analytical Methods. Mass Spectrom Purif Tech. 2016; 2(101). doi: 10.4172/2469-9861.1000e101

59. Heidari A. Yoctosecond Quantitative Structure-Activity Relationship (QSAR) and Quantitative Structure-Property Relationship (QSPR) under Synchrotron Radiations Studies for Prediction of Solubility of Anti-Cancer Nano Drugs in Aqueous Solutions Using Genetic Function Approximation (GFA) Algorithm. Insight Pharm Res. 2016; 1(1).

60. Heidari A. Cancer Risk Prediction and Assessment in Human Cells under Synchrotron Radiations Using Quantitative Structure Activity Relationship (QSAR) and Quantitative Structure Properties Relationship (QSPR) Studies. Int J Clin Med Imaging. 2016; 3(516).
61. Heidari A. A Novel Approach to Biology. Electronic J Biol. 2016; 12(4).

62. Heidari A. Innovative Biomedical Equipment's for Diagnosis and Treatment. J Bioengineer \& Biomedical Sci. 2016; 6(2).

63. Heidari A. Integrating Precision Cancer Medicine into Healthcare. Medicare Reimbursement Changes and the Practice of Oncology: Trends in Oncology Medicine and Practices. J Oncol Med \& Pract. 2016; 1(2).

64. Heidari A. Promoting Convergence in Biomedical and Biomaterials Sciences and Silk Proteins for Biomedical and Biomaterials Applications: An Introduction to Materials in Medicine and Bioengineering Perspectives. J Bioengineer \& Biomedical Sci. 2016; 6(3). doi: 10.4172/21559538.1000e126

65. Heidari A. X-Ray Fluorescence and X-Ray Diffraction Analysis on Discrete Element Modeling of Nano Powder Metallurgy Processes in Optimal Container Design. J Powder Metall Min. 2017; 6(1). doi: 10.4172/21689806.1000e136

66. Heidari A. Biomolecular Spectroscopy and Dynamics of Nano-Sized Molecules and Clusters as Cross-Linking-Induced Anti-Cancer and Immune-Oncology Nano Drugs Delivery in DNA/RNA of Human Cancer Cells' Membranes under Synchrotron Radiations: A Payload-Based Perspective. Arch Chem Res. 2017; 1(2). doi: 10.21767/2572-4657.100011

67. Heidari A. Deficiencies in Repair of Double-Standard DNA/RNA-Binding Molecules Identified in Many Types of Solid and Liquid Tumors Oncology in Human Body for Advancing Cancer Immunotherapy Using Computer Simulations and Data Analysis. J Appl Bioinforma Comput Biol. 2017; 6(1). doi: 10.4172/2329-9533.1000e104

68. Heidari A. Electronic Coupling among the Five Nanomolecules Shuts Down Quantum Tunneling in the Presence and Absence of an Applied Magnetic Field for Indication of the Dimer or other Provide Different Influences on the Magnetic Behavior of Single Molecular Magnets (SMMs) as Qubits for Quantum Computing. Glob J Res Rev. 2017; 4(2). doi: 10.21767/2393-8854.100019

69. Heidari A. Polymorphism in Nano-Sized Graphene Ligand-Induced Transformation of Au38-xAgx/xCux(SPh-tBu)24 to Au36-xAgx/xCux(SPhtBu)24 ( $x=1-12)$ Nanomolecules for Synthesis of Au144-xAgx/xCux[(SR)60. (SC4)60. (SC6)60. (SC12)60. (PET)60. (p-MBA)60. (F)60. (Cl)60. (Br)60. (I)60. (At)60. (Uus)60 and (SC6H13)60] Nano Clusters as Anti-Cancer Nano Drugs. J Nanomater Mol Nanotechnol. 2017; 6(3). doi: 10.4172/2324-8777.1000e109

70. Heidari A. Biomedical Resource Oncology and Data Mining to Enable Resource Discovery in Medical. Medicinal. Clinical. Pharmaceutical. Chemical and Translational Research and Their Applications in Cancer Research. Int J Biomed Data Min. 2017; 6(03). doi: 10.4172/20904924.1000e103

71. Heidari A. Study of Synthesis. Pharmacokinetics. Pharmacodynamics. Dosing.Stability. Safety and Efficacy of Olympiadane Nanomolecules as Agent for Cancer Enzymotherapy. Immunotherapy. Chemotherapy. Radiotherapy. Hormone Therapy and Targeted Therapy under Synchrotorn Radiation. J Dev Drugs. 2017; 6(154). doi: 10.4172/2329$6631.1000 \mathrm{e} 154$

72. Heidari A. A Novel Approach to Future Horizon of Top Seven Biomedical Research Topics to Watch in 2017: Alzheimer's. Ebola. Hypersomnia. Human Immunodeficiency Virus (HIV). Tuberculosis (TB). Microbiome/ Antibiotic Resistance and Endovascular Stroke. J Bioengineer \& Biomedical Sci. 2017; 7(127). doi: 10.4172/2155-9538.1000e127

73. Heidari A. Opinion on Computational Fluid Dynamics(CFD)Technique. Fluid Mech Open Acc. 2017; 4(157). doi: 10.4172/2476-2296.1000157

74. Heidari A. Concurrent Diagnosis of Oncology Influence Outcomes in Emergency General Surgery for Colorectal Cancer and Multiple Sclerosis (MS) Treatment Using Magnetic Resonance Imaging (MRI) and Au329(SR)84 Au329-xAgx(SR)84. Au144(SR)60. Au68(SR)36. Au30(SR)18. Au102(SPh)44. Au38(SPh)24. Au38(SC2H4Ph)24. Au21S(SAdm)15. Au36(pMBA)24 and Au25(pMBA)18 Nano Clusters. J Surgery Emerg Med. 2017; 1(2): 1.

75. Heidari A. Developmental Cell Biology in Adult Stem Cells Death and Autophagy to Trigger a Preventive Allergic Reaction to Common Airborne Allergens under Synchrotron Radiation Using Nanotechnology for Therapeutic Goals in Particular Allergy Shots (Immunotherapy). Cell Biol (Henderson. NV). 2017; 6(1). doi: 10.4172/2324-9293.1000e117 
76. Heidari A. Changing Metal Powder Characteristics for Elimination of the Heavy Metals Toxicity and Diseases in Disruption of Extracellular Matrix (ECM) Proteins Adjustment in Cancer Metastases Induced by Osteosarcoma. Chondrosarcoma. Carcinoid. Carcinoma. Ewing's Sarcoma. Fibrosarcoma and Secondary Hematopoietic Solid or Soft Tissue Tumors. J Powder Metall Min. 2017; 6(1)70. Doi: 10.4172/2168-9806.1000170

77. Heidari A. Nanomedicine-Based Combination Anti-Cancer Therapy between Nucleic Acids and Anti-Cancer Nano Drugs in Covalent Nano Drugs Delivery Systems for Selective Imaging and Treatment of Human Brain Tumors Using Hyaluronic Acid. Alguronic Acid and Sodium Hyaluronate as Anti-Cancer Nano Drugs and Nucleic Acids Delivery under Synchrotron Radiation. Am J Drug Deliv. 2017; 5(2). doi: 10.21767/2321-547X.1000016

78. Heidari A. Clinical Trials of Dendritic Cell Therapies for Cancer Exposing Vulnerabilities in Human Cancer Cells' Metabolism and Metabolomics: New Discoveries. Unique Features Inform New Therapeutic Opportunities. Biotech's Bumpy Road to the Market and Elucidating the Biochemical Programs that Support Cancer Initiation and Progression. J Biol Med Science. 2017; 1(103).

79. Heidari A. The Design Graphene-Based Nanosheets as a New Nanomaterial in Anti-Cancer Therapy and Delivery of Chemotherapeutics and Biological Nano Drugs for Liposomal Anti-Cancer Nano Drugs and Gene Delivery. $\mathrm{Br}$ Biomed Bull. 2017; 5(305). doi: 10.21767/2471-9897.1000305

80. Heidari A. Integrative Approach to Biological Networks for Emerging Roles of Proteomics. Genomics and Transcriptomics in the Discovery and Validation of Human Colorectal Cancer Biomarkers from DNA/RNA Sequencing Data under Synchrotron Radiation. Transcriptomics. 2017; 5(20): e117. doi: 10.4172/2329-8936.1000e117

81. Heidari A. Elimination of the Heavy Metals Toxicity and Diseases in Disruption of Extracellular Matrix (ECM) Proteins and Cell Adhesion Intelligent Nanomolecules Adjustment in Cancer Metastases Using Metalloenzymes and under Synchrotron Radiation. Lett Health Biol Sci. 2017; 2(2): 1-4. doi: 10.15436/2475-6245.17.019

82. Heidari A. Treatment of Breast Cancer Brain Metastases through a Targeted Nanomolecule Drug Delivery System Based on Dopamine Functionalized Multi-Wall Carbon Nanotubes (MWCNTs) Coated with Nano Graphene Oxide (GO) and Protonated Polyaniline (PANI) in Situ During the Polymerization of Aniline Autogenic Nanoparticles for the Delivery of Anti-Cancer Nano Drugs under Synchrotron Radiation. Br J Res. 2017; 4(3): 16. doi: 10.21767/2394-3718.100016

83. Heidari A. Sedative. Analgesic and Ultrasound-Mediated Gastrointestinal Nano Drugs Delivery for Gastrointestinal Endoscopic Procedure. Nano Drug-Induced Gastrointestinal Disorders and Nano Drug Treatment of Gastric Acidity. Res Rep Gastroenterol. 2017; 1(1).

84. Heidari A. Synthesis, Pharmacokinetics, Pharmacodynamics, Dosing, Stability, Safety and Efficacy of Orphan Nano Drugs to Treat High Cholesterol and Related Conditions and to Prevent Cardiovascular Disease Under Synchrotron Radiation. J Pharm Sci Emerg Drugs. 2017; 5(1). doi: 10.4172/2380-9477.1000e104

85. Heidari A. Non Linear Compact Proton Synchrotrons to Improve Human Cancer Cells and Tissues Treatments and Diagnostics through Particle Therapy Accelerators with Monochromatic Microbeams. J Cell Biol Mol Sci. 2017; 2(1): 1-5. doi: 10.15436/2471-5891.17.1631

86. Heidari A. Design of Targeted Metal Chelation Therapeutics Nanocapsules as Colloidal Carriers and Blood-Brain Barrier (BBB) Translocation to Targeted Deliver Anti-Cancer Nano Drugs into the Human Brain to Treat Alzheimer's Disease under Synchrotron Radiation. J Nanotechnol Material Sci. 2017; 4(2): 1-5. doi: 10.15436/2377-1372.17.1591

87. Gobato R, Heidari A. Calculations Using Quantum Chemistry for Inorganic Molecule Simulation BeLi2SeSi. Science Journal of Analytical Chemistry. 2017; 5(6): 76-85. doi: 10.11648/j.ajqcms.20170203.12

88. Heidari A. Different High-Resolution Simulations of Medical. Medicinal. Clinical. Pharmaceutical and Therapeutics Oncology of Human Lung Cancer Translational Anti-Cancer Nano Drugs Delivery Treatment Process under Synchrotron and X-Ray Radiations. J Med Oncol. 2017; 1(1): 1.

89. Heidari A. A Modern Ethnomedicinal Technique for Transformation. Prevention and Treatment of Human Malignant Gliomas Tumors into Human Benign Gliomas Tumors under Synchrotron Radiation. Am J Ethnomed. 2017; 4(1:10).
90. Heidari A. Active Targeted Nanoparticles for Anti-Cancer Nano Drugs Delivery across the Blood-Brain Barrier for Human Brain Cancer Treatment. Multiple Sclerosis (MS) and Alzheimer's Diseases Using Chemical Modifications of Anti-Cancer Nano Drugs or Drug-Nanoparticles through Zika Virus (ZIKV) Nanocarriers under Synchrotron Radiation. J Med Chem Toxicol. 2017; 2(3): 1-5. 2017. doi: 10.15436/2575-808X.17.1594

91. Heidari A. Investigation of Medical. Medicinal. Clinical and Pharmaceutical Applications of Estradiol. Mestranol (Norlutin). Norethindrone (NET). Norethisterone Acetate (NETA). Norethisterone Enanthate (NETE) and Testosterone Nanoparticles as Biological Imaging. Cell Labeling. AntiMicrobial Agents and Anti-Cancer Nano Drugs in Nanomedicines Based Drug Delivery Systems for Anti-Cancer Targeting and Treatment. Parana Journal of Science and Education (PJSE). 2017; 3(4): 10-19.

92. Heidari A. A Comparative Computational and Experimental Study on Different Vibrational Biospectroscopy Methods. Techniques and Applications for Human Cancer Cells in Tumor Tissues Simulation. Modeling. Research. Diagnosis and Treatment. Open J Anal Bioanal Chem. 2017; 1(1): 014-020.

93. Heidari A. Combination of DNA/RNA Ligands and Linear/Non-Linear Visible-Synchrotron Radiation-Driven N-Doped Ordered Mesoporous Cadmium Oxide (CdO) Nanoparticles Photocatalysts Channels Resulted in an Interesting Synergistic Effect Enhancing Catalytic Anti-Cancer Activity. Enz Eng. 2017; 6(1). doi: 10.4172/2329-6674.1000160

94. Heidari A. Modern Approaches in Designing Ferritin. Ferritin Light Chain. Transferrin. Beta-2 Transferrin and Bacterioferritin-Based Anti-Cancer Nano Drugs Encapsulating Nanosphere as DNA-Binding Proteins from Starved Cells (DPS). Mod Appro Drug Des. 2017; 1(1): 000504 . doi: 10.31031/MADD.2017.01.000504

95. Heidari A. Potency of Human Interferon $\beta-1 \mathrm{a}$ and Human Interferon $\beta-1 \mathrm{~b}$ in Enzymotherapy. Immunotherapy. Chemotherapy. Radiotherapy. Hormone Therapy and Targeted Therapy of Encephalomyelitis Disseminate/Multiple Sclerosis (MS) and Hepatitis A. B. C. D. E. F and G Virus Enter and Targets Liver Cells. J Proteomics Enzymol. 2017; 6(1). doi: 10.4172/2470-1289.1000e109

96. Heidari A. Transport Therapeutic Active Targeting of Human Brain Tumors Enable Anti-Cancer Nanodrugs Delivery across the Blood-Brain Barrier (BBB) to Treat Brain Diseases Using Nanoparticles and Nanocarriers under Synchrotron Radiation. J Pharm Pharmaceutics. 2017; 4(2): 1-5. doi: 10.15436/2377-1313.17.034

97. Heidari A, Brown C. Combinatorial Therapeutic Approaches to DNA/RNA and Benzylpenicillin (Penicillin G). Fluoxetine Hydrochloride (Prozac and Sarafem). Propofol (Diprivan). Acetylsalicylic Acid (ASA) (Aspirin). Naproxen Sodium (Aleve and Naprosyn) and Dextromethamphetamine Nanocapsules with Surface Conjugated DNA/RNA to Targeted Nano Drugs for Enhanced Anti-Cancer Efficacy and Targeted Cancer Therapy Using Nano Drugs Delivery Systems. Ann Adv Chem. 2017; 1(2): 061-069. doi: 10.29328/journal.aac.1001008

98. Heidari A. High-Resolution Simulations of Human Brain Cancer Translational Nano Drugs Delivery Treatment Process under Synchrotron Radiation. J Transl Res. 2017; 1(1): 1-3.

99. Heidari A. Investigation of Anti-Cancer Nano Drugs' Effects' Trend on Human Pancreas Cancer Cells and Tissues Prevention. Diagnosis and Treatment Process under Synchrotron and X-Ray Radiations with the Passage of Time Using Mathematica. Current Trends Anal Bioanal Chem. 2017; 1(1): 36-41.

100. Heidari A. Pros and Cons Controversy on Molecular Imaging and Dynamics of Double-Standard DNA/RNA of Human Preserving Stem Cells-Binding Nano Molecules with Androgens/Anabolic Steroids (AAS) or Testosterone Derivatives through Tracking of Helium-4 Nucleus (Alpha Particle) Using Synchrotron Radiation. Arch Biotechnol Biomed. 2017; 1(1) 067-0100. doi: 10.29328/journal.hjb.1001007

101. Heidari A. Visualizing Metabolic Changes in Probing Human Cancer Cells and Tissues Metabolism Using Vivo 1H or Proton NMR. 13C NMR. 15N NMR and 31P NMR Spectroscopy and Self-Organizing Maps under Synchrotron Radiation. SOJ Mater Sci Eng. 2017; 5(2): 1-6.

102. Heidari A. Cavity Ring-Down Spectroscopy (CRDS). Circular Dichroism Spectroscopy. Cold Vapour Atomic Fluorescence Spectroscopy and Correlation Spectroscopy Comparative Study on Malignant and Benign Human Cancer Cells and Tissues with the Passage of Time under Synchrotron Radiation. Enliven: Challenges Cancer Detect Ther. 2017; 4(2): e001. 
103. Heidari A. Laser Spectroscopy. Laser-Induced Breakdown Spectroscopy and Laser-Induced Plasma Spectroscopy Comparative Study on Malignant and Benign Human Cancer Cells and Tissues with the Passage of Time under Synchrotron Radiation. Int J Hepatol Gastroenterol. 2017; 3(4): 079-084.

104. Heidari A. Time-Resolved Spectroscopy and Time-Stretch Spectroscopy Comparative Study on Malignant and Benign Human Cancer Cells and Tissues with the Passage of Time under Synchrotron Radiation. Enliven: Pharmacovigilance and Drug Safety. 2017; 4(2): e001.

105. Heidari A. Overview of the Role of Vitamins in Reducing Negative Effect of Decapeptyl (Triptorelin Acetate or Pamoate Salts) on Prostate Cancer Cells and Tissues in Prostate Cancer Treatment Process through Transformation of Malignant Prostate Tumors into Benign Prostate Tumors under Synchrotron Radiation. Open J Anal Bioanal Chem. 2017; 1(1): 021-026.

106. Heidari A. Electron Phenomenological Spectroscopy. Electron Paramagnetic Resonance (EPR) Spectroscopy and Electron Spin Resonance (ESR) Spectroscopy Comparative Study on Malignant and Benign Human Cancer Cells and Tissues with the Passage of Time under Synchrotron Radiation. Austin J Anal Pharm Chem. 2017; 4(3): 1091.

107. Heidari A. Therapeutic Nanomedicine Different High-Resolution Experimental Images and Computational Simulations for Human Brain Cancer Cells and Tissues Using Nanocarriers Deliver DNA/RNA to Brain Tumors under Synchrotron Radiation with the Passage of Time Using Mathematica and MATLAB. Madridge J Nano Tech. Sci. 2(2): 77-83.

108. Heidari A. A Consensus and Prospective Study on Restoring Cadmium Oxide $(\mathrm{CdO})$ Nanoparticles Sensitivity in Recurrent Ovarian Cancer by Extending the Cadmium Oxide (CdO) Nanoparticles-Free Interval Using Synchrotron Radiation Therapy as Antibody-Drug Conjugate for the Treatment of Limited-Stage Small Cell Diverse Epithelial Cancers. Cancer Clin Res Rep. 2017; 1(2): e001.

109. Heidari A. A Novel and Modern Experimental Imaging and Spectroscopy Comparative Study on Malignant and Benign Human Cancer Cells and Tissues with the Passage of Time under White Synchrotron Radiation. Cancer Sci Res Open Access. 2017; 4(2): 1-8. doi: 10.15226/csroa.2017.00137

110. Heidari A. Different High-Resolution Simulations of Medical. Medicinal. Clinical. Pharmaceutical and Therapeutics Oncology of Human Breast Cancer Translational Nano Drugs Delivery Treatment Process under Synchrotron and X-Ray Radiations. J Oral Cancer Res. 2017; 1(1): $12-17$.

111. Heidari A. Vibrational Decihertz $(\mathrm{dHz})$. Centihertz $(\mathrm{cHz})$. Millihertz $(\mathrm{mHz})$. Microhertz $(\mu \mathrm{Hz})$. Nanohertz $(\mathrm{nHz})$. Picohertz $(\mathrm{pHz})$. Femtohertz $(\mathrm{fHz})$. Attohertz $(\mathrm{aHz})$. Zeptohertz $(\mathrm{zHz})$ and Yoctohertz $(\mathrm{yHz})$ Imaging and Spectroscopy Comparative Study on Malignant and Benign Human Cancer Cells and Tissues under Synchrotron Radiation. International Journal of Biomedicine. 2017; 7(4). 335-340. doi: 10.21103/Article7(4)_IA1

112. Heidari A. Force Spectroscopy and Fluorescence Spectroscopy Comparative Study on Malignant and Benign Human Cancer Cells and Tissues with the Passage of Time under Synchrotron Radiation. EC Cancer. 2017; 2(5): 239-246.

113. Heidari A. Photoacoustic Spectroscopy. Photoemission Spectroscopy and Photothermal Spectroscopy Comparative Study on Malignant and Benign Human Cancer Cells and Tissues with the Passage of Time under Synchrotron Radiation. BAOJ Cancer Res Ther. 2017; 3(3): 045-052.

114. Heidari A. J-Spectroscopy. Exchange Spectroscopy (EXSY). Nuclear Overhauser Effect Spectroscopy (NOESY) and Total Correlation Spectroscopy (TOCSY) Comparative Study on Malignant and Benign Human Cancer Cells and Tissues under Synchrotron Radiation. EMS Eng Sci J. 2017; 1(2): 006-013.

115. Heidari A. Neutron Spin Echo Spectroscopy and Spin Noise Spectroscopy Comparative Study on Malignant and Benign Human Cancer Cells and Tissues with the Passage of Time under Synchrotron Radiation. Int $J$ Biopharm Sci. 2017; 1(1): 103-107. doi: 10.31021/ijbs.20181103

116. Heidari A. Vibrational Decahertz (daHz). Hectohertz (hHz). Kilohertz $(\mathrm{kHz})$. Megahertz (MHz). Gigahertz (GHz). Terahertz (THz). Petahertz (PHz). Exahertz (EHz). Zettahertz $(\mathrm{ZHz})$ and Yottahertz $(\mathrm{YHz})$ Imaging and Spectroscopy Comparative Study on Malignant and Benign Human Cancer Cells and Tissues under Synchrotron Radiation. Madridge J Anal Sci Instrum. 2017; 2(1): 41-46.
117. Heidari A. Two-Dimensional Infrared Correlation Spectroscopy. Linear Two-Dimensional Infrared Spectroscopy and Non-Linear TwoDimensional Infrared Spectroscopy Comparative Study on Malignant and Benign Human Cancer Cells and Tissues under Synchrotron Radiation with the Passage of Time. J Mater Sci Nanotechnol. 2018; 6(1): 101.

118. Heidari A. Fourier Transform Infrared (FTIR) Spectroscopy. Near-Infrared Spectroscopy (NIRS) and Mid-Infrared Spectroscopy (MIRS) Comparative Study on Malignant and Benign Human Cancer Cells and Tissues under Synchrotron Radiation with the Passage of Time. Int J Nanotechnol Nanomed. 2018; 3(1): 1-6.

119. Heidari A. Infrared Photo Dissociation Spectroscopy and Infrared Correlation Table Spectroscopy Comparative Study on Malignant and Benign Human Cancer Cells and Tissues under Synchrotron Radiation with the Passage of Time. Austin Pharmacol Pharm. 2018; 3(1): 1011.

120. Heidari A. Novel and Transcendental Prevention. Diagnosis and Treatment Strategies for Investigation of Interaction among Human Blood Cancer Cells. Tissues. Tumors and Metastases with Synchrotron Radiation under Anti-Cancer Nano Drugs Delivery Efficacy Using MATLAB Modeling and Simulation. Madridge J Nov Drug Res. 2017; 1(1): 18-24.

121. Heidari A. Comparative Study on Malignant and Benign Human Cancer Cells and Tissues with the Passage of Time under Synchrotron Radiation. Open Access J Trans Med Res. 2018; 2(1): 00026-00032. doi: 10.15406/ oajtmr.2018.02.00026

122. Gobato MRR, Gobato R, Heidari A. Planting of Jaboticaba Trees for Landscape Repair of Degraded Area. Landscape Architecture and Regional Planning. 2018; 3(1): 1-9. doi: 10.11648/j.larp.20180301.11

123. Heidari A. Fluorescence Spectroscopy. Phosphorescence Spectroscopy and Luminescence Spectroscopy Comparative Study on Malignant and Benign Human Cancer Cells and Tissues under Synchrotron Radiation with the Passage of Time. SM J Clin. Med. Imaging. 2018; 4(1): 1018.

124. Heidari A. Nuclear Inelastic Scattering Spectroscopy (NISS) and Nuclear Inelastic Absorption Spectroscopy (NIAS) Comparative Study on Malignant and Benign Human Cancer Cells and Tissues under Synchrotron Radiation. Int J Pharm Sci. 2018; 2(1): 1-14. doi: 10.19070/2332-2756$180008 \mathrm{e}$

125. Heidari A. X-Ray Diffraction (XRD). Powder X-Ray Diffraction (PXRD) and Energy-Dispersive X-Ray Diffraction (EDXRD) Comparative Study on Malignant and Benign Human Cancer Cells and Tissues under Synchrotron Radiation. J Oncol Res. 2018; 2(1): 1-14.

126. Heidari A. Correlation Two-Dimensional Nuclear Magnetic Reso $\neg n a n c e$ (NMR) (2D-NMR) (COSY) Imaging and Spectrosco $\neg$ py Comparative Study on Malignant and Benign Human Cancer Cells and Tissues under Synchrotron Radiation. Arch Biotechnol Biomed. 2017; 1(1): 067-0100. doi: 10.29328/journal.hjb.1001007

127. Heidari A. Thermal Spectroscopy. Photothermal Spectroscopy. Thermal Microspectroscopy. Photothermal Microspectroscopy. Thermal Macrospectroscopy and Photothermal Macrospectroscopy Comparative Study on Malignant and Benign Human Cancer Cells and Tissues with the Passage of Time under Synchrotron Radiation. SM J Biometrics Biostat. 2018; 3(1): 1024.

128. Heidari A. A Modern and Comprehensive Experimental Biospectroscopic Comparative Study on Human Common Cancers' Cells. Tissues and Tumors before and after Synchrotron Radiation Therapy. Open Acc J Oncol Med. 2018; 1(1).

129. Heidari A. Heteronuclear Correlation Experiments such as Heteronuclear Single-Quantum Correlation Spectroscopy (HSQC). Heteronuclear Multiple-Quantum Correlation Spectroscopy (HMQC) and Heteronuclear Multiple-Bond Correlation Spectroscopy (HMBC) Comparative Study on Malignant and Benign Human Endocrinology and Thyroid Cancer Cells and Tissues under Synchrotron Radiation. J Endocrinol Thyroid Res. 2018; 3(1): 555603. doi: 10.19080/JETR.2018.03.555604

130. Heidari A. Nuclear Resonance Vibrational Spectroscopy (NRVS). Nuclear Inelastic Scattering Spectroscopy (NISS). Nuclear Inelastic Absorption Spectroscopy (NIAS) and Nuclear Resonant Inelastic X-Ray Scattering Spectroscopy (NRIXSS) Comparative Study on Malignant and Benign Human Cancer Cells and Tissues under Synchrotron Radiation. Int J Bioorg Chem Mol Biol. 2018; 6(1e): 1-5. Doi: 10.19070/2332-2756-180008e 
131. Heidari A. A Novel and Modern Experimental Approach to Vibrational Circular Dichroism Spectroscopy and Video Spectroscopy Comparative Study on Malignant and Benign Human Cancer Cells and Tissues with the Passage of Time under White and Monochromatic Synchrotron Radiation. Glob J Endocrinol Metab. 2018; 1(3): 000514-000519.

132. Heidari A. Pros and Cons Controversy on Heteronuclear Correlation Experiments such as Heteronuclear Single-Quantum Correlation Spectroscopy (HSQC). Heteronuclear Multiple-Quantum Correlation Spectroscopy (HMQC) and Heteronuclear Multiple-Bond Correlation Spectroscopy (HMBC) Comparative Study on Malignant and Benign Human Cancer Cells and Tissues under Synchrotron Radiation. EMS Pharma J. 2018; 1(1): 002. doi: 10.19080/JETR.2018.03.555604

133. Heidari A. A Modern Comparative and Comprehensive Experimental Biospectroscopic Study on Different Types of Infrared Spectroscopy of Malignant and Benign Human Cancer Cells and Tissues with the Passage of Time under Synchrotron Radiation. J Analyt Molecul Tech. 2018; 3(1): 8.

134. Heidari A. Investigation of Cancer Types Using Synchrotron Technology for Proton Beam Therapy: An Experimental Biospectroscopic Comparative Study. European Modern Studies Journal. 2018; 2(1): 13-29.

135. Heidari A. Saturated Spectroscopy and Unsaturated Spectroscopy Comparative Study on Malignant and Benign Human Cancer Cells and Tissues with the Passage of Time under Synchrotron Radiation. Imaging J Clin Medical Sci. 2018; 5(1): 001-007. doi: 10.17352/2455-8702.000036

136. A. Heidari. Small-Angle Neutron Scattering (SANS) and Wide-Angle $X-$ Ray Diffraction (WAXD) Comparative Study on Malignant and Benign Human Cancer Cells and Tissues under Synchrotron Radiation. Int J Bioorg Chem Mol Biol. 2018; 6(2e): 1-6. doi: 10.19070/2332-2756-180009e

137. Heidari A. Investigation of Bladder Cancer. Breast Cancer. Colorectal Cancer. Endometrial Cancer. Kidney Cancer. Leukemia. Liver. Lung Cancer. Melanoma. Non-Hodgkin Lymphoma. Pancreatic Cancer. Prostate Cancer. Thyroid Cancer and Non-Melanoma Skin Cancer Using Synchrotron Technology for Proton Beam Therapy: An Experimental Biospectroscopic Comparative Study. Ther Res Skin Dis. 2018; 1(1).

138. Heidari A. Attenuated Total Reflectance Fourier Transform Infrared (ATRFTIR) Spectroscopy. Micro-Attenuated Total Reflectance Fourier Transform Infrared (Micro-ATR-FTIR) Spectroscopy and Macro-Attenuated Total Reflectance Fourier Transform Infrared (Macro-ATR-FTIR) Spectroscopy Comparative Study on Malignant and Benign Human Cancer Cells and Tissues under Synchrotron Radiation with the Passage of Time. International Journal of Chemistry Papers. 2018; 2(1): 1-12. doi: 10.1021/la700713g

139. Heidari A. Mössbauer Spectroscopy. Mössbauer Emission Spectroscopy and 57Fe Mössbauer Spectroscopy Comparative Study on Malignant and Benign Human Cancer Cells and Tissues under Synchrotron Radiation. Acta Scientific Cancer Biology. 2018; 2(3): 17-20. 2018.

140. Heidari A. Comparative Study on Malignant and Benign Human Cancer Cells and Tissues under Synchrotron Radiation with the Passage of Time. Organic \& Medicinal Chem IJ. 2018; 6(1): 555676. doi: 10.15406/ oajtmr.2018.02.00026

141. Heidari A. Correlation Spectroscopy. Exclusive Correlation Spectroscopy and Total Correlation Spectroscopy Comparative Study on Malignant and Benign Human AIDS-Related Cancers Cells and Tissues with the Passage of Time under Synchrotron Radiation. Int J Bioanal Biomed. 2018; 2(1): 001-007.
142. Heidari A. Biomedical Instrumentation and Applications of Biospectroscopic Methods and Techniques in Malignant and Benign Human Cancer Cells and Tissues Studies under Synchrotron Radiation and Anti-Cancer Nano Drugs Delivery. Am J Nanotechnol Nanomed. 2018; 1(1): 001-009.

143. Heidari A. Vivo $1 \mathrm{H}$ or Proton NMR. $13 \mathrm{C}$ NMR. $15 \mathrm{~N}$ NMR and 31P NMR Spectroscopy Comparative Study on Malignant and Benign Human Cancer Cells and Tissues under Synchrotron Radiation. Ann Biomet Biostat. 2018; 1(1): 1001.

144. Heidari A. Grazing-Incidence Small-Angle Neutron Scattering (GISANS) and Grazing-Incidence X-Ray Diffraction (GIXD) Comparative Study on Malignant and Benign Human Cancer Cells. Tissues and Tumors under Synchrotron Radiation. Ann Cardiovasc Surg. 2018; 1(2): 1006.

145. Heidari A. Adsorption Isotherms and Kinetics of Multi-Walled Carbon Nanotubes (MWCNTs). Boron Nitride Nanotubes (BNNTs). Amorphous Boron Nitride Nanotubes (a-BNNTs) and Hexagonal Boron Nitride Nanotubes (h-BNNTs) for Eliminating Carcinoma. Sarcoma. Lymphoma. Leukemia. Germ Cell Tumor and Blastoma Cancer Cells and Tissues. Clin Med Rev Case Rep. 2018; 5(201). doi: 10.23937/2378-3656/1410201

146. Heidari A. Correlation Spectroscopy (COSY). Exclusive Correlation Spectroscopy (ECOSY). Total Correlation Spectroscopy (TOCSY). Incredible Natural-Abundance Double-Quantum Transfer Experiment (INADEQUATE). Heteronuclear Single-Quantum Correlation Spectroscopy (HSQC). Heteronuclear Multiple-Bond Correlation Spectroscopy (HMBC). Nuclear Overhauser Effect Spectroscopy (NOESY) and Rotating Frame Nuclear Overhauser Effect Spectroscopy (ROESY) Comparative Study on Malignant and Benign Human Cancer Cells and Tissues under Synchrotron Radiation. Acta Scientific Pharmaceutical Sciences. 2018; 2(5): 30-35. doi: 10.19080/OMCIJ.2018.06.555695

147. Heidari A. Small-Angle X-Ray Scattering (SAXS). Ultra-Small Angle X-Ray Scattering (USAXS). Fluctuation X-Ray Scattering (FXS). Wide-Angle XRay Scattering (WAXS). Grazing-Incidence Small-Angle X-Ray Scattering (GISAXS). Grazing-Incidence Wide-Angle X-Ray Scattering (GIWAXS). Small-Angle Neutron Scattering (SANS). Grazing-Incidence Small-Angle Neutron Scattering (GISANS). X-Ray Diffraction (XRD). Powder X-Ray Diffraction (PXRD). Wide-Angle X-Ray Diffraction (WAXD). GrazingIncidence X-Ray Diffraction (GIXD) and Energy-Dispersive X-Ray Diffraction (EDXRD) Comparative Study on Malignant and Benign Human Cancer Cells and Tissues under Synchrotron Radiation. Oncol Res Rev. 2018; 1(1): 1-10. doi: 10.15761/ORR.1000104

148. Heidari A. Pump-Probe Spectroscopy and Transient Grating Spectroscopy Comparative Study on Malignant and Benign Human Cancer Cells and Tissues with the Passage of Time under Synchrotron Radiation. Adv Material Sci Engg. 2018; 2 (1): 1-7.

149. Heidari A. Grazing-Incidence Small-Angle X-Ray Scattering (GISAXS) and Grazing-Incidence Wide-Angle X-Ray Scattering (GIWAXS) Comparative Study on Malignant and Benign Human Cancer Cells and Tissues under Synchrotron Radiation. Insights Pharmacol Pharm Sci. 2018; 1(1): 1-8.

150. Heidari A. Acoustic Spectroscopy. Acoustic Resonance Spectroscopy and Auger Spectroscopy Comparative Study on Anti-Cancer Nano Drugs Delivery in Malignant and Benign Human Cancer Cells and Tissues with the Passage of Time under Synchrotron Radiation. Nanosci Technol. 2018; 5(1): 1-9. 